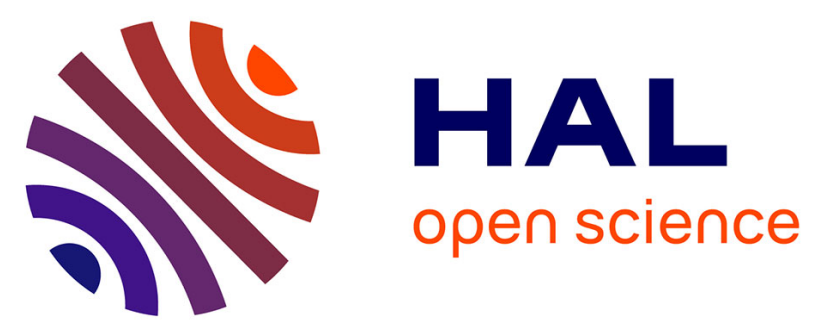

\title{
Determining displacement and strain maps immune from aliasing effect with the grid method
}

Frédéric Sur, Benoît Blaysat, Michel Grediac

\section{To cite this version:}

Frédéric Sur, Benoît Blaysat, Michel Grediac. Determining displacement and strain maps immune from aliasing effect with the grid method. Optics and Lasers in Engineering, 2016, 86, pp.317-328. 10.1016/j.optlaseng.2016.06.010 . hal-01352868

\section{HAL Id: hal-01352868 \\ https://hal.science/hal-01352868}

Submitted on 10 Aug 2016

HAL is a multi-disciplinary open access archive for the deposit and dissemination of scientific research documents, whether they are published or not. The documents may come from teaching and research institutions in France or abroad, or from public or private research centers.
L'archive ouverte pluridisciplinaire HAL, est destinée au dépôt et à la diffusion de documents scientifiques de niveau recherche, publiés ou non, émanant des établissements d'enseignement et de recherche français ou étrangers, des laboratoires publics ou privés. 


\title{
Determining displacement and strain maps immune from aliasing effect with the grid method
}

\author{
Frédéric Sur, Benoît Blaysat’, Michel GrédiaC ${ }^{\ddagger}$
}

\begin{abstract}
Spatial aliasing may affect methods based on grid image processing to retrieve displacement and strain maps in experimental mechanics. Such methods aim at estimating these maps on the surface of a specimen subjected to a loading test. Aliasing, which is often not noticeable to the naked eye in the grid images, may give spurious fringes in the strain maps. This paper presents an analysis of aliasing in this context and provides the reader with simple guidelines to minimize the effect of aliasing on strain maps extracted from grid images.
\end{abstract}

Keywords: aliasing, spectrum folding, grid method, displacement and strain maps.

This is the author-manuscript version of

F. Sur, B. Blaysat, M. Grédiac. Determining displacement and strain maps immune from aliasing effect with the grid method. Optics and Lasers in Engineering, vol. 86, p. 317-328, Elsevier, 2016.

DOI: $10.1016 / j \cdot$ optlaseng.2016.06.010

\footnotetext{
*Laboratoire Lorrain de Recherche en Informatique et ses Applications (LORIA), UMR 7503, Université de Lorraine, CNRS, INRIA projet Magrit, Campus Scientifique BP 239, 54506 Vandœuvre-lès-Nancy Cedex, France.

†Institut Pascal, UMR 6602, Clermont Université, Université Blaise Pascal, CNRS, BP 10448, 63000 ClermontFerrand, France.

${ }^{\ddagger}$ Institut Pascal, UMR 6602, Clermont Université, Université Blaise Pascal, CNRS, BP 10448, 63000 ClermontFerrand, France.
} 


\section{Introduction}

Non-interferometric full-field measurement techniques are spreading more and more in the experimental mechanics community. Digital image correlation (DIC) [24] is certainly the most popular one thanks to its versatility. Indeed, this technique does not only work with randomly marked patterns, which are the most common ones because they are quite easy to deposit on the external surface of specimens, but also with regular patterns such as dots or grids. In this latter case, however, another possibility consists in processing the corresponding grid images using spectral methods to obtain in-plane displacement and strain distributions. These spectral methods present several advantages: for instance, metrological performances can be quantified with closed-form expressions [9], no optimization algorithm is needed, and the retrieved strain maps are not the result of an interpolation between remote points where the measurement is performed. Nevertheless, a phenomenon often occurs when acquiring images of grids with a digital camera: the presence of parasitic fringes, that is, quasi-periodic patterns superposed to the expected grid image. Although these fringes may be hardly noticeable in the acquired images, they cause spurious patterns in the estimated displacement maps, and mainly in the strain maps. This phenomenon is due to the fact that regular patterns are shot by a camera sensor, which is made of an array of pixels. The signal is thus sampled, and aliasing may occur if the Nyquist condition is not satisfied in the PetersenMiddleton sampling theorem [17] (a generalization to images of the 1D Nyquist-Shannon sampling theorem [16]).

It is worth mentioning that these parasitic fringes are of low amplitude and even often imperceptible to the naked eye in grid images. The impact of these parasitic fringes on displacement and strain maps is, however, potentially significant, these maps being retrieved from actual changes between images which are generally of low amplitude. This is precisely the case in many situations of deformed materials or structures studied with full-field measurement techniques. This phenomenon also occurs when local strain increments are to be calculated, as in plasticity. Indeed, strain increments between consecutive images may be small even though the strain itself is significant. As a consequence, strain increment maps are also potentially affected by spurious fringes. The effect of aliasing on strain and strain increment maps is illustrated in Figure 1.

A first solution to avoid these fringes is to defocus the lens in order to blur the acquired images. This approach limits aliasing by smoothing out the high-frequency components of the imaged grid, thus enforcing the Nyquist sampling condition. Nevertheless, defocusing reduces the sharpness and the contrast of the acquired image, which causes the signal-to-noise ratio in final strain maps to decrease according to the discussion in $[9,20]$. It has been shown, in particular, that the standard deviation of the noise in final displacement and strain maps increases when the contrast decreases. While the anti-aliasing filter (an optical low-pass filter) which equips many digital cameras is supposed to eliminate aliasing, it is often not present in high-end cameras, and it does not completely eliminate aliasing, since a trade-off between sharpness and Nyquist condition must be reached. Another potential solution, effective in some cases, consists in eliminating aliasing through a post-processing step, for instance by applying a suitable notch filter in the frequency domain, as explained in [11] in the case of strain maps. Designing such notch filters for eliminating so-called pseudo-periodic noise in images is the aim of several papers in the image processing or microscopy community $[1,2,12,14,19,27,28]$, but it seems that the procedures enabling the automatic design of such filters are less efficient in the case of displacement and strain maps, mainly because these maps feature statistical properties which are different from those of natural images [21]. In addition, some information is irremediably lost in such a procedure. Note finally that parasitic fringes in final 

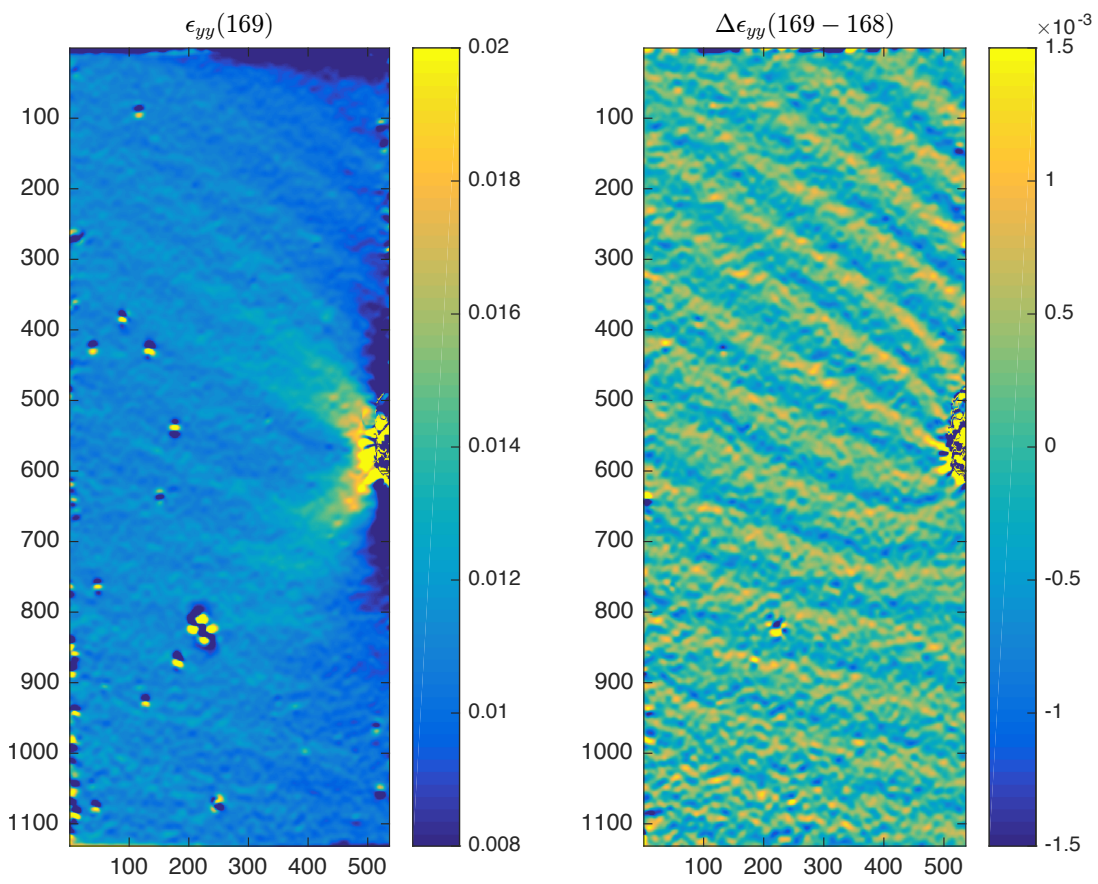

Figure 1: Strain and strain increments measured on an open-hole specimen during a tensile test. Spurious fringes can be seen. They are caused by aliasing, which affects the grid image. This experiment is discussed in Section 4.2.

strain maps obtained from grid images may be due to other reasons than aliasing, for instance:

- regular grid defects due to printing. Processing the images using the method proposed in $[4,5]$ enables one to get rid of this effect;

- unsuitable window and/or settings when using the windowed Fourier transform to process grid images, as explained in [22].

These last two cases are not addressed here: we only consider the case of fringes impairing grid images caused by aliasing. These fringes propagate up to the final displacement or strain maps when processing the grid images.

In this context, the aim of this paper is to propose and analyze a simple procedure to avoid this undesirable phenomenon: rotating the grid with respect to the camera sensor by a certain angle, whose determination will be detailed. The benefit, compared to the aforementioned solutions, is to keep the highest contrast in the grid images (and thus the lowest noise level in the displacement and strain maps), and to avoid the awkward design of notch filters in the Fourier domain.

The paper is organized as follows: Section 2 briefly recalls the basics of the spectral methods used classically to process grid images. The continuous (in the sense that the space variable is continuous) and discrete (the space variable is sampled) spectra of grid images are then discussed in Section 3, with a special emphasis put on an important consequence of sampling, namely aliasing. We propose in Section 4 a numerical example, which shows that rotating the grid by a certain angle allows us to 
avoid aliasing, and thus the appearance of spurious fringes in strain maps. The effectiveness of the method is illustrated by real strain maps obtained with two grids deposited on a specimen subjected to a tensile test (one is rotated, the other is not). Section 5 discusses the optimum rotation angle between the imaged grid and the pixel grid. Some demonstrations and reminders are gathered in separate annexes.

\section{Grid methods in experimental mechanics}

\subsection{Retrieving displacement and strain maps from grid images using spectral methods}

In experimental mechanics, grids have long been used to measure in-plane displacement and strain components by processing deformed and nondeformed grid images. A detailed review being given in [10], we only recall here the main available techniques, and we describe in more detail the one used in the present work. Grids being deformed, they can no more be considered as a perfect periodic signal but instead as a phase-modulated signal. The first step is thus to estimate phase modulations. Basically, two spectral techniques have been proposed to deduce displacement and strain maps from grid images, namely geometric phase analysis (GPA), and methods based on windowed Fourier analysis at the nominal frequency of the grid. With GPA, the phase is estimated by taking the Fourier transform of images of a regular pattern, filtering the corresponding spectra with a notch filter centered around the spike of the principal frequency of the pattern, performing the inverse Fourier transform to retrieve the corresponding phase distributions and unwrapping the obtained results. This technique was inspired by procedures employed when processing fringes obtained in interferometry, as explained in the seminal papers $[25,26]$ for instance. It was then adapted to lattice images observed at the microscopic scale in [13], and finally extended at the macroscopic scale on grids directly deposited on specimens in $[6,7]$. The windowed version of the Fourier transform can also be employed to process grid images, as proposed in [23]. The region surrounding the spikes of the principal frequencies is considered in $[6,7]$, but only the nominal frequency of the grid is taken into account in [23], which dramatically accelerates the calculations. Recent applications mainly deal with the technique presented in [23]. In addition, it has been shown that closed-form expressions for various metrological parameters can be elaborated in this case $[9,10,20]$, which is important to eventually give the measurement uncertainty of the obtained results, as defined in [15]. For all these reasons, we employ the windowed Fourier transform (WFT) with the nominal frequency of the grid to process the grid images discussed in this paper. The analysis of aliasing proposed here is, however, still valid when the grid images are processed with other spectral methods, for instance GPA [6,7]. Indeed and as we shall see, the procedure presented here permits to mitigate the effect of aliasing on the useful spectrum component. It is independent of the spectral method used to process the grids and retrieve displacement and strain maps. In contrast, methods that process grid images globally (for instance digital image correlation [24], or the methods described in [3] or [8]), instead of focusing on a limited part of the spectrum, are potentially still affected by aliasing. 


\subsection{Notations and reminder}

In this article, the Fourier transform of any integrable $2 \mathrm{D}$ function $f(x, y)$ is denoted by $\mathcal{F}(f)(u, v)$ and is defined as:

$$
\mathcal{F}(f)(u, v)=\iint_{\mathbb{R}^{2}} f(x, y) e^{-2 i \pi(x u+y v)} \mathrm{d} x \mathrm{~d} y
$$

Similarly, the windowed Fourier transform is defined at any point $(\xi, \eta)$ by

$$
\mathcal{F}_{w}(f)(\xi, \eta, u, v)=\iint_{\mathbb{R}^{2}} f(x, y) w(x-\xi, y-\eta) e^{-2 i \pi(x u+y v)} \mathrm{d} x \mathrm{~d} y
$$

where $w$ is the analysis window. This is an isotropic and positive function integrating to 1 . The Gaussian window defined by

$$
w(x, y)=\frac{1}{2 \pi \sigma^{2}} e^{\left(-\frac{x^{2}+y^{2}}{2 \sigma^{2}}\right)}
$$

is employed here, where $\sigma$ denotes its standard deviation. We keep the same notations for the Fourier transform of tempered distributions, which allows us to deal with Dirac's delta or constant functions.

Both the 2D Fourier transform and its windowed version are covariant with any rotation of the image domain. More precisely, for any $\theta \in \mathbb{R}$, if $f_{R}(x, y)=f(x \cos (\theta)+y \sin (\theta),-x \sin (\theta)+y \cos (\theta))$, we have

$$
\mathcal{F}\left(f_{R}\right)(u, v)=\mathcal{F}(f)(u \cos (\theta)+v \sin (\theta),-u \sin (\theta)+v \cos (\theta))
$$

and

$$
\begin{aligned}
\mathcal{F}_{w}\left(f_{R}\right)(\xi, \eta, u, v)=\mathcal{F}_{w}(f)(\xi \cos (\theta)+\eta \sin (\theta),-\xi \sin (\theta)+\eta \cos (\theta), & \\
& u \cos (\theta)+v \sin (\theta),-u \sin (\theta)+v \cos (\theta))
\end{aligned}
$$

The proof of this property is reminded in Appendix A.

The modulus of any complex number $z$ is denoted by $|z|$, and if $z \neq 0$, its argument is denoted by $\arg (z)$.

The Dirac distribution centered at $(a, b) \in \mathbb{R}^{2}$ is denoted by $\delta_{a, b}$. The convolution of two integrable functions or of two tempered distributions is denoted by $f * g$. The relation $\mathcal{F}(f * g)=$ $\mathcal{F}(f) \mathcal{F}(g)$ holds.

\subsection{Estimating phases through windowed Fourier transform}

We consider that we have a set of grid images shot during a mechanical test. The bidimensional signal corresponding to each image is denoted by $s(x, y)$.

Let us assume for a while that grids, that is, orthogonal sets of parallel lines, are aligned with the 2D coordinate axes. Following [4], a continuous grid can be modeled as follows:

$$
s(x, y)=\frac{A(x, y)}{2}\left(2+\gamma \cdot \ell\left(2 \pi f x+\phi_{1}(x, y)\right)+\gamma \cdot \ell\left(2 \pi f y+\phi_{2}(x, y)\right)\right)
$$

where

- $A(x, y)>0$ is the global field illumination, which may slightly vary across the field of view; 
- $\gamma \in[0,1]$ is the contrast of the oscillatory pattern;

- the line profile $\ell$ is a $2 \pi$-periodic real function with a peak amplitude equal to 1 and average value 0 ;

- $f$ is the nominal frequency of the carrier, the grid pitch being $p=1 / f$;

- $\phi_{1}(x, y)$ and $\phi_{2}(x, y)$ are the carrier phase modulations due to specimen surface displacements along the $x$ and $y$-axes respectively. In practice, their spatial derivatives are very small with respect to $2 \pi f$.

In real grid images, the amplitude of the signal is not exactly twice that of each of the two line networks considered separately. Although this does not affect the conclusion of the calculation performed below, we discuss this point in Section 4.

As explained above, we consider here the nominal frequency of the grid to process the grid images with the WFT recalled in (2). The value of the WFT is thus calculated twice at any pixel $(\xi, \eta)$ of the image, once for $(f, 0)$ and once for $(0, f)$, where $f$ is equal to the nominal frequency of the grid (referred to as the principal harmonic in the remainder of the paper). This frequency is assumed to be known a priori. The standard deviation $\sigma$ in (3) is equal to $1 / f$ as requested in [20]. We obtain two complex numbers defined at each pixel $(\xi, \eta): \mathcal{F}_{w}(s)(\xi, \eta, f, 0)$ and $\mathcal{F}_{w}(s)(\xi, \eta, 0, f)$. It is generally assumed that the arguments of these complex numbers are merely the two sought phases $\phi_{1}$ and $\phi_{2}$. More rigorously, these quantities are, up to first order approximations, the sought phases convolved by the window $w$ (up to an additive constant, see [20]). The convolution slightly blurs the actual distributions. Note that these phases have to be unwrapped. In the case of a rotated grid, the phases must be brought back along the horizontal and vertical axis through the opposite rotation:

$$
\left\{\begin{array}{l}
\Phi_{1}(x, y)=\phi_{1}(x, y) \cos (\theta)-\phi_{2}(x, y) \sin (\theta) \\
\Phi_{2}(x, y)=\phi_{1}(x, y) \sin (\theta)+\phi_{2}(x, y) \cos (\theta)
\end{array}\right.
$$

Both phases $\Phi_{1}\left(\Phi_{2}\right.$, respectively), taken after and before deformation, are subtracted and finally multiplied by $-\frac{p}{2 \pi}$ to deduce the in-plane displacement at any pixel along the horizontal axis (the vertical axis, respectively). Note that the movement of the physical points shall be taken into account to get rid of the grid defects, as proposed in [4]. The interested reader is referred to the review paper [10] for more details. The linearized strain components are finally obtained by calculating the symmetric part of the displacement gradient.

The remainder of the paper examines how seeking the displacement and strain distributions may be disturbed by aliasing, which potentially affects the grid images. Without loss of generality, the discussion of aliasing is led with the Fourier transform. It is indeed still valid when the signal is localized through windowing.

\section{Spectrum of a grid}

This section discusses the spectrum of a grid, within the model of (6). Let $\ell(x)=\sum_{k \in \mathbb{Z}} d_{k} e^{i k x}$ be the Fourier expansion of the $2 \pi$-periodic $\ell$ function. Since $\ell$ is a 0 -mean real function, $d_{0}=0$ and for any $k, d_{k}$ is the complex conjugate of $d_{-k}$. A pure sine is theoretically the ideal line profile [9] (in which case for any $k \neq \pm 1, d_{k}=0$ ), but it is not possible to print such grids in practice. 


\subsection{Continuous spectrum}

The Fourier transform of $s$ writes (see Appendix B):

$$
\begin{aligned}
\mathcal{F}(s)(u, v)=\mathcal{F}(A)(u, v)+\frac{\gamma}{2} \sum_{k \in \mathbb{Z}} d_{k} \mathcal{F}(A) * \mathcal{F}\left(e^{i k \phi_{1}}\right) & (u-k f, v) \\
& +\frac{\gamma}{2} \sum_{k \in \mathbb{Z}} d_{k} \mathcal{F}(A) * \mathcal{F}\left(e^{i k \phi_{2}}\right)(u, v-k f)
\end{aligned}
$$

If $A$ can be considered to be constant, then $\mathcal{F}(A)=A \delta_{0,0}$ and (8) simplifies into the following equation:

$$
\mathcal{F}(s)(u, v)=A \delta_{0,0}+\frac{\gamma A}{2} \sum_{k \in \mathbb{Z}} d_{k} \mathcal{F}\left(e^{i k \phi_{1}}\right)(u-k f, v)+\frac{\gamma A}{2} \sum_{k \in \mathbb{Z}} d_{k} \mathcal{F}\left(e^{i k \phi_{2}}\right)(u, v-k f)
$$

Assuming that both partial derivatives of $\phi_{l}(l=1$ or 2$)$ are bounded, Proposition C.1 (in Appendix $\mathrm{C}$ ) proves that, for any $k$, the support of $\mathcal{F}\left(e^{i k \phi_{l}}\right)$ is included in

$$
\left[-\frac{|k|}{p} M_{l}^{x}, \frac{|k|}{p} M_{l}^{x}\right] \times\left[-\frac{|k|}{p} M_{l}^{y}, \frac{|k|}{p} M_{l}^{y}\right]
$$

where $M_{l}^{x}=(p / 2 \pi) \max \left(\left|\partial \phi_{l} / \partial x\right|\right)$ and $M_{l}^{y}=(p / 2 \pi) \max \left(\left|\partial \phi_{l} / \partial y\right|\right)$. Note that with these notations, $M_{l}^{x}$ and $M_{l}^{y}$ can be considered as bounds for the strain components, under the hypothesis that the grid on the specimen is perfectly aligned with the pixel grid in the reference state, the phase modulation being produced only by the deformation of the specimen.

This means that a grid image spectrum is made of a central spike caused by the $\mathcal{F}(A)$ term and separated spikes centered at multiples of the frequency $f$ along the horizontal and vertical axes of the spectrum. These spikes are caused by the $\mathcal{F}\left(e^{i k \phi_{1}}\right)$ and $\mathcal{F}\left(e^{i k \phi_{2}}\right)$ terms, and by the harmonics of the line profile $\ell$. These spikes are therefore mostly included in

$$
\left[-\frac{|k|}{p} M_{1}^{x}+k f, \frac{|k|}{p} M_{1}^{x}+k f\right] \times\left[-\frac{|k|}{p} M_{1}^{y}, \frac{|k|}{p} M_{1}^{y}\right]
$$

along the horizontal direction, and

$$
\left[-\frac{|k|}{p} M_{2}^{x}, \frac{|k|}{p} M_{2}^{x}\right] \times\left[-\frac{|k|}{p} M_{2}^{y}+k f, \frac{|k|}{p} M_{2}^{y}+k f\right]
$$

along the vertical direction. We write "mostly" because of the blur caused by the convolution of the Fourier transform $\mathcal{F}(A)$ of the illumination field in (8), in addition to the approximate calculation in the stationary phase method. Moreover, this property also means that the support of the spikes is all the narrower as the upper bounds of the partial derivatives of the phase are small, and as $k \geq 1$ is small. Figure 2 illustrates a typical spectrum of a grid.

Since a spectrum of a rotated grid is simply the rotated spectrum of a grid aligned with the pixels (see Section 2.2 above), the preceding discussion adapts easily to rotated grids. 


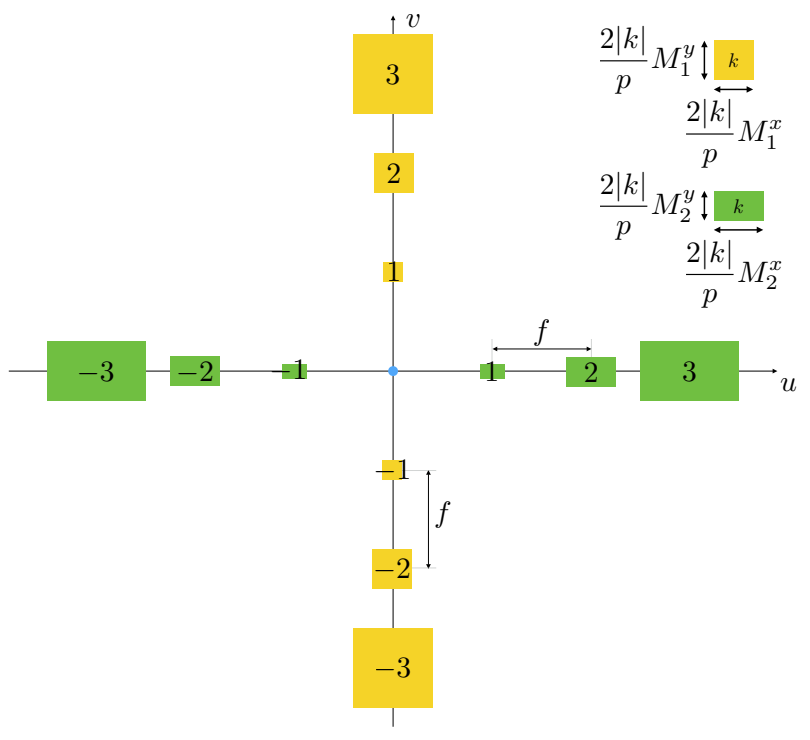

Figure 2: An illustration of the continuous spectrum of a grid, supposed here to generate only three pairs of harmonics along each axis.

\subsection{Discrete spectrum}

Digital sensors give sampled information. Assuming square pixels, which is a sound assumption in most real cameras, Nyquist-Shannon sampling theorem states that the continuous signal is well sampled if and only if its spectrum is supported by the box $\left[-f_{x}^{s} / 2, f_{x}^{s} / 2\right] \times\left[-f_{y}^{s} / 2, f_{y}^{s} / 2\right]$, where $f_{x}^{s}, f_{y}^{s}$ are the sampling frequencies along both discretization directions. In what follows, $f_{x}^{s}=f_{y}^{s}=f^{s}=1$ pixel $^{-1}$. Note that the case of non-square pixels is still tackled by Peterson-Middleton theorem, the localization condition becoming that the continuous spectrum must be included in the so-called reciprocal cell.

Let us assume that the camera gives a discrete image $\bar{s}$ of size $M \times N(M$ and $N$ are assumed to be even), which samples the continuous $s$ discussed in the preceding section. The question is: how the spectrum of the discrete image (obtained with the discrete Fourier transform of $\bar{s}$ ) is related to the spectrum of the continuous image given by (8)?

Let $c_{m, n}$ be the discrete Fourier coefficient (with $-M / 2 \leq m \leq M / 2-1$ and $-N / 2 \leq n \leq$ $N / 2-1)$ of $\bar{s}$, that is

$$
c_{m, n}=\sum_{k=-M / 2}^{M / 2-1} \sum_{l=-N / 2}^{N / 2-1} \bar{s}(k, l) e^{-2 i \pi(k m / M+\ln / N)}
$$

Since the complex exponential function is $2 i \pi$-periodic, $c_{m, n}$ can be considered as an $(M, N)$-periodic two-dimensional series along both indices $M$ and $N$.

With these notations, for any $m, n$ such that $-M / 2 \leq m \leq M / 2-1$ and $-N / 2 \leq n \leq N / 2-1$, $c_{m, n}$ is the Fourier coefficient associated with a sinusoidal component of respective frequencies $|m|$ and $|n|$ cycles-per-image along horizontal and vertical axes. In this unity, frequencies are bounded by 
$\max (M, N) / 2$. Normalized frequencies $|m| / M$ and $|n| / N$ are in cycles-per-pixel, and are bounded by $1 / 2$.

The support of the continuous image $s$ such that $\bar{s}(k, l)=s(k, l)$ is supposed to be included in $[-M / 2, M / 2-1] \times[-N / 2, N / 2-1]$ (that is, $s=0$ outside of this box). From Appendix D, for any $m, n$ such that $-M / 2 \leq m \leq M / 2-1$ and $-N / 2 \leq n \leq N / 2-1$, the Fourier coefficient $c_{m, n}$ satisfies:

$$
\begin{aligned}
c_{m, n} & =\sum_{(k, l) \in \mathbb{Z}^{2}} \mathcal{F}(s)\left(\frac{m}{M}+k, \frac{n}{N}+l\right) \\
& =\mathcal{F}(s)\left(\frac{m}{M}, \frac{n}{N}\right)+\sum_{(k, l) \in \mathbb{Z}^{2} \backslash\{0,0\}} \mathcal{F}(s)\left(\frac{m}{M}+k, \frac{n}{N}+l\right)
\end{aligned}
$$

This means that the discrete spectrum (i.e., the set of the $M \times N$ coefficients $c_{m, n}$ ) corresponds to the discretized continuous spectrum $\mathcal{F}(s)$, to which are added copies of the discretized $\mathcal{F}(s)$ translated by an integer value (the so-called aliases, or folded components). Aliasing thus manifests itself by impairing the spectrum of the sampled image by the components of the continuous spectrum lying outside $[-1 / 2,1 / 2] \times[-1 / 2,1 / 2]$, thus breaking the Nyquist condition.

\subsection{Impact on phase estimation from grid images}

Aliasing causes spurious fringes in the phase maps, which propagate to the displacement and mainly strain maps through the process described in Section 2.3. Since these fringes are not identical after and before deformation, they do not vanish when subtracting the phases between the current and the reference states. In this section, we focus on the impact of aliasing on the phase maps.

The method described in [4, 10] (after [23]) for finding the phase is based on the estimation of $\mathcal{F}(s)(f, 0)$ and $\mathcal{F}(s)(0, f)$, and the method described in [6, 7] (after [26, 25]) is based on the estimation of $\mathcal{F}(s)$ in neighborhoods of $(f, 0)$ and $(0, f)$ (in order to obtain estimations of $\mathcal{F}\left(e^{i k \phi_{1}}\right)$ and $\mathcal{F}\left(e^{i k \phi_{2}}\right)$ in $\left.(8)\right)$. As mentioned above, these methods are actually based on windowed Fourier analysis, but the discussion of aliasing in the previous section is still valid locally.

Nevertheless, the input datum (the imaged grid) is acquired by a digital sensor and is discrete. The continuous spectrum $\mathcal{F}(s)$ is not available, and the calculations are led with the discrete Fourier coefficients $c_{m, n}$. As a consequence of the discussion in Section 3.2, aliasing impairs the retrieved phase maps as soon as the Fourier coefficients of interest are impaired by the terms $\mathcal{F}(s)\left(\frac{m}{M}+k, \frac{n}{N}+l\right)$, with $(k, l) \neq(0,0)$. Even if the "spike" around $(f, 0)$ or $(0, f)$ is not aliased (or, in other words, if it is fully included in $[-1 / 2,1 / 2] \times[-1 / 2,1 / 2]$ ), it may be covered by aliased harmonic spikes outside of this box in the original spectrum of $s$. In this case, aliasing appears in the retrieved phases and subsequently in the estimated displacement and strain maps.

In the case of grid images aligned with the pixel grid of the sensor, this means that the $k$-th harmonics of the line profile give aliasing as soon as $k \geq f^{s} /(2 f)$. For instance, with a typical value of $f=1 / 5$ pixel $^{-1}$, meaning that the pattern pitch is encoded on five pixels, harmonics such that $k \geq 3$ are aliased and thus potentially cover the spikes of interest. With a 45-degree rotation, harmonics such that $k \geq \sqrt{2} f^{s} /(2 f)$ (thus $\left.k \geq 4\right)$ are aliased for the same $f / f^{s}$ value. Note that decreasing $f$ (i.e., increasing the grid pitch $p=1 / f$, thus the number of pixels imaged over a period) also decreases the number of aliased harmonics. This solution is often not possible in practice because of the trade-off to be satisfied between the pixel density of the sensor and the field of view of the lens. Figure 3 shows, however, that aliasing may be got rid of by rotating the 

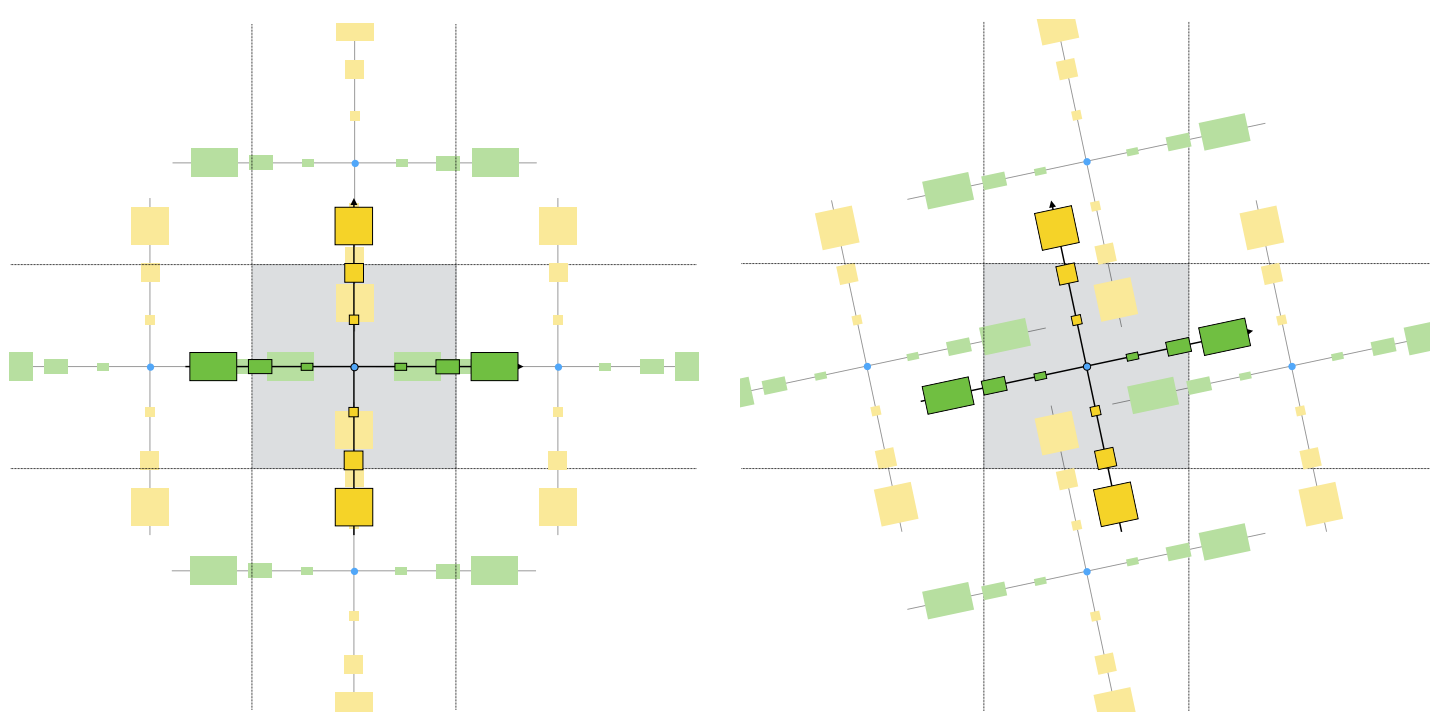

Figure 3: An illustration of the discrete spectrum of a grid image, with an aligned-to-pixel grid (on the left) and a rotated grid (on the right). With the notations of (15), the translated copies of $\mathcal{F}(s)$ for $(k, l) \in\{(0,1),(0,-1),(1,0),(-1,0)\}$ are shown in a light shade, superposed to the non-translated $\mathcal{F}(s)$ in the central, gray square. The discrete Fourier coefficients $c_{m, n}$ are the sum of all the components superposed in the gray square. We can see in the left sketch that the principal harmonic is covered by an aliased spike. On the contrary, the right sketch shows that turning the grid prevents the aliased spikes from covering the principal harmonic.

grid instead of increasing the grid pitch. According to the property reminded in Section 2.2 above, a rotation of the grid image is indeed equivalent to a rotation of the spectrum $\mathcal{F}(s)$. It can be noted that the rotation angle $\theta$ and the indices $\left(m^{*}, n^{*}\right)$ of the Fourier coefficient corresponding to the principal harmonic satisfy $\tan (\theta)=\left(m^{*} N\right) /\left(n^{*} M\right)$. The resulting procedure is investigated in the following section.

\section{Illustrative examples}

We propose herein two illustrative examples: a numerical experiment and a real-case study. We demonstrate that rotating the grid image with respect to the pixel grid significantly reduces the effect of aliasing on the estimation of the displacement and strain maps.

\subsection{Numerical experiment}

This section provides the reader with a numerical illustration of aliasing in the context of displacement and strain map estimation. Grid images are simulated according to the model of (6), with a constant illumination $A=2^{11}$, a contrast $\gamma=0.9$, a line profile $\ell(x)=\sin ^{7}(x)$ (chosen to generate realistic sharp lines and several harmonics), and $p=1 / f$ the grid pitch. Such grid images mimic the output of a standard 12-bit camera. Concerning the phase modulations, we choose $\phi_{1}=0$, and $\phi_{2}$ 

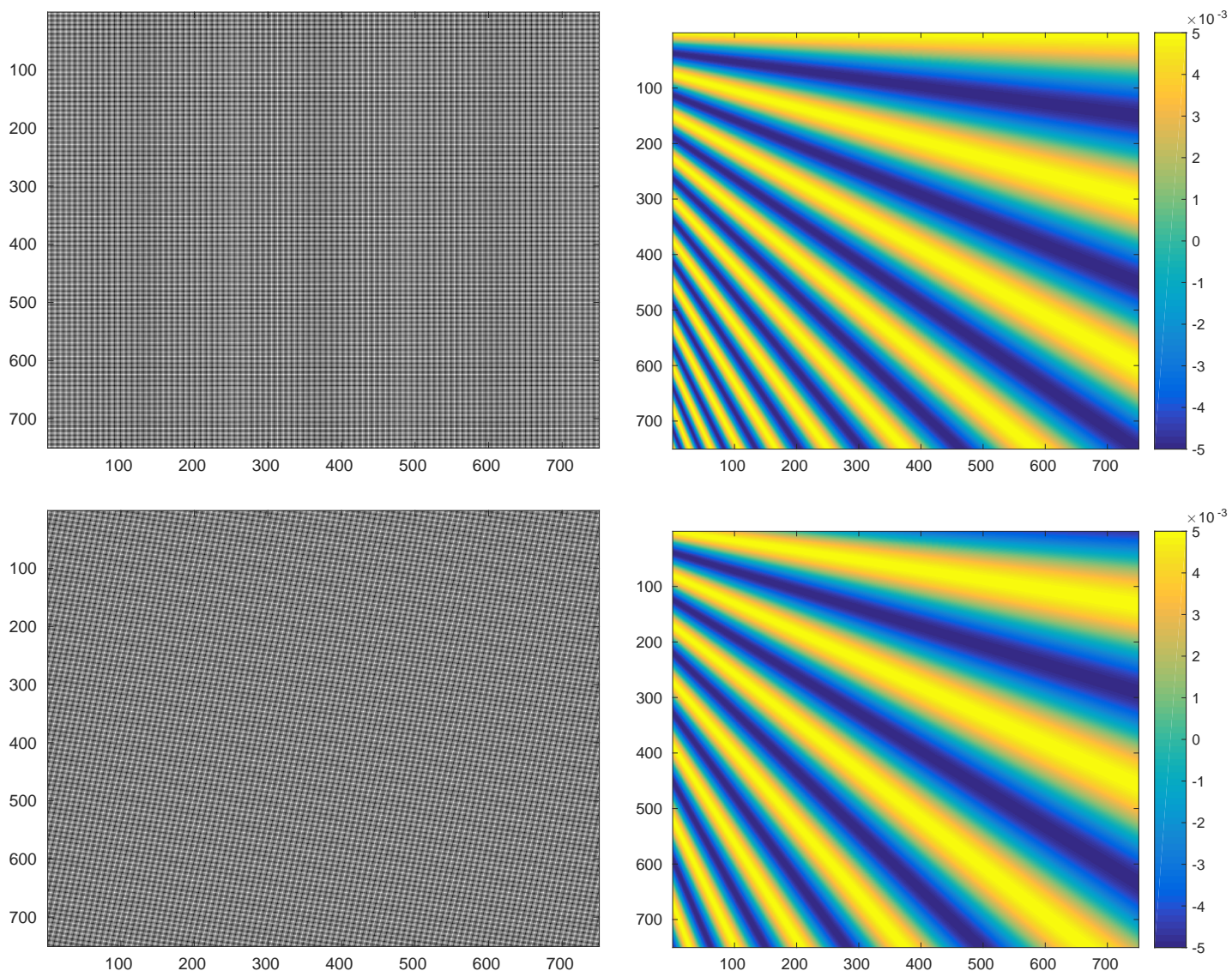

Figure 4: Synthetic grid. Upper row: aligned-to-pixel grid. Bottom row: rotated grid. On the left: grid image. On the right: derivative of the imposed phase modulation.

such that $\partial \phi_{2} / \partial y$ is a sine wave along the $x$-direction, with a linearly varying period along $y$. The amplitude of this sine wave is constant, equal to $5 \cdot 10^{-3} \mathrm{pixel}^{-1}$. While (6) gives a continuous aligned-to-pixel grid, a clockwise rotation of 10 degrees around the origin of the pixel coordinates gives the rotated grid. Both grids are then sampled to produce an $N \times N$ image. Here, the size of an image is chosen to linearly increase with the pattern pitch $p$ in order to simulate an increased image resolution of a given field of view. We set $N=200(p-4)$ so that the minimum pattern pitch $(p=5)$ gives a $200 \times 200$ image. This procedure permits to generate images of fixed grids, their resolution increasing with the pitch $p$. Figure 4 illustrates the generated grids and the corresponding maps of $\partial \phi_{2} / \partial y$, for $p=7.75$ pixels. Note that the phase derivatives are brought back in the same coordinate axis through the procedure described in Section 2.3 above.

The $\ell$ function being odd, its Fourier coefficients $d_{k}$ are such that $d_{k}=0$ for any odd integer $k$, and the calculation shows that $d_{k} \neq 0$ only for $k= \pm 1, \pm 3, \pm 5, \pm 7$. The spectrum of the alignedto-pixel grid image, as shown in Figure 5, complies with the theory of Section 3. Spikes can be seen on the horizontal axis in spite that $\phi_{x}=0$ because of sampling, which results in a slight phase 

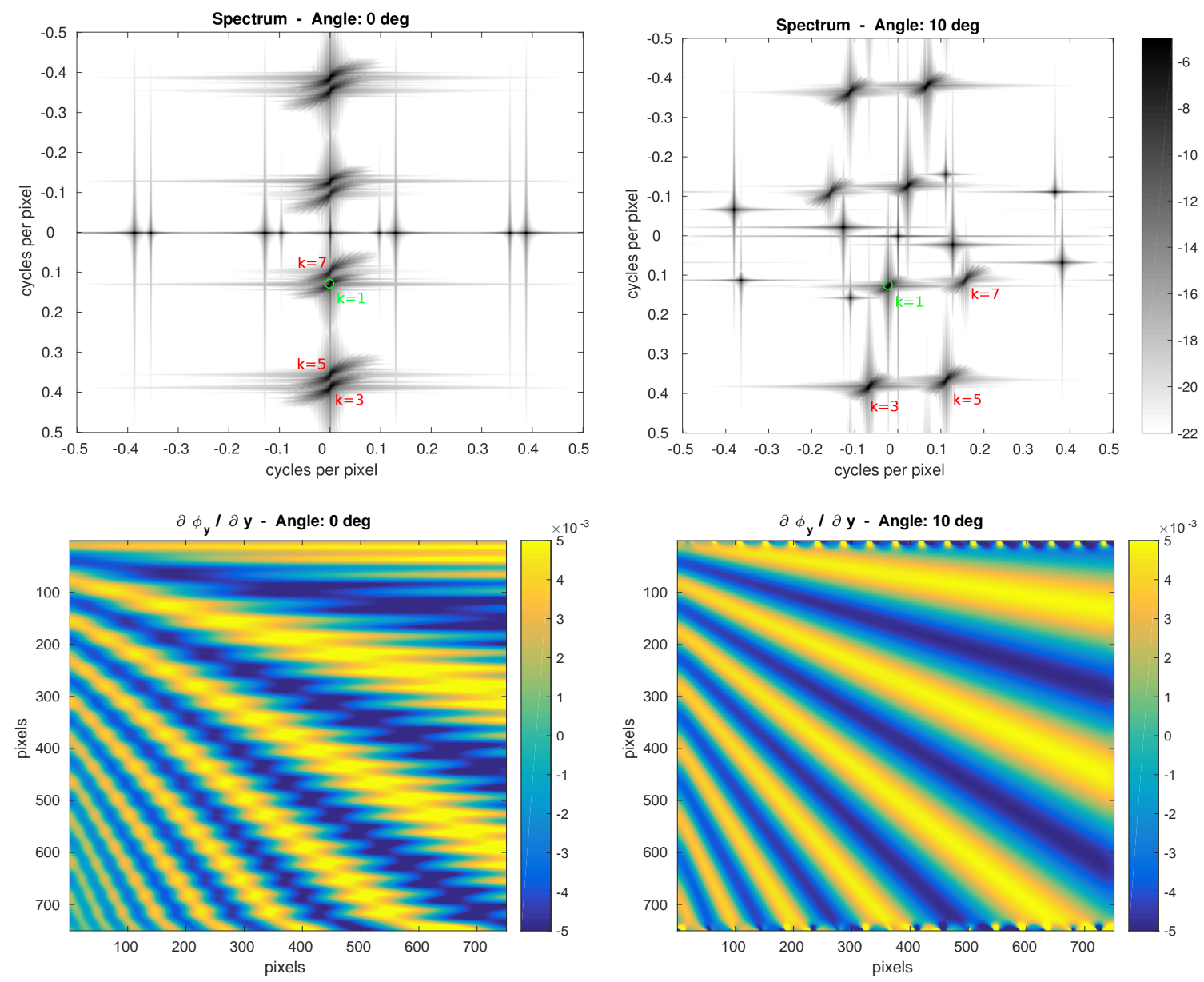

Figure 5: Synthetic grid. On the top: spectrum of the aligned-to-pixel grid image (on the left) and of the rotated grid image (on the right). Frequencies are in cycles per image. The principal harmonic is circled in green. At the bottom: retrieved phase derivative from the aligned-to-pixel grid (on the left) and from the rotated grid (on the right). These figures should be compared to the ground truth presented in Figure 4. Aliasing in the aligned-to-pixel grid dramatically transfers to the phase derivative maps as parasitic spurious fringes.

modulation. Along the vertical axis, four "spikes" can be seen at a distance of $|k| / p=0.129 \cdot k$ (for $k= \pm 1, \pm 3$ ) cycles per image from the frequency origin, and four spikes correspond to the folding of the spikes expected for $k= \pm 5, \pm 7$, for which $|k / p|>0.5$. Folded spikes can be found respectively at a distance of $1-0.129 \cdot 5=0.355$ and $1-0.129 \cdot 7=0.097$ of the frequency origin. Since the folded spike corresponding to $k=7$ overlaps the principal harmonic at $1 / p=0.129$, aliasing is expected to impair the retrieved phase maps. Concerning the rotated grid image, spectrum folding can still be noted. Nevertheless, a visual inspection shows that, in this case, folded spikes do not overlap 
the principal harmonic. Aliasing is thus expected to be far less noticeable in the retrieved phase maps. Figure 5 also shows the estimated phase derivative maps $\partial \phi_{y} / \partial y$. While aliasing indeed produces strong fringes as expected when the phase is estimated from the aligned-to-pixel grid, no such spurious phenomenon can be seen when the estimation is performed on the rotated grid.

A companion video (file video_aliasing_synth.avi) shows the evolution of the retrieved phase derivative with such synthetic grid images. Both the spectrum and the retrieved phase derivative are shown for grid pitch values increasing from 5 to 10 pixels, for the aligned-to-pixel and the rotated grids. Aliased spikes can be seen. They propagate to the retrieved phase derivative map as soon as they cover the principal harmonic, marked with a green circle. In this case, dramatic interferences can be noted. Rotating the grid adequately (here, with a 15-degree angle) permits to shift away the aliased spikes outside the principal harmonic in the spectrum. In this case, no spurious fringe can be seen in the retrieved phase derivative.

It is interesting to note that, when an aliased spikes (corresponding to the $k$-th harmonic) is perfectly superposed to the principal harmonic, the Fourier coefficient $c_{f M, 0}$ corresponding to the normalized frequency $(f, 0)$ is given by:

$$
\frac{\gamma A}{2}\left(d_{1} \mathcal{F}\left(e^{i \phi_{1}}\right)(0,0)+d_{k} \mathcal{F}\left(e^{i k \phi_{1}}\right)(0,0)\right)
$$

(we use the simplified expression (9), knowing that the non-aliased harmonics vanish at $(f, 0)$ in this expression).

As explained in the introduction, the estimation of the phase $\phi_{2}$ is performed by taking the argument of the coefficient given in (16). Moreover, $\mathcal{F}\left(e^{i k \phi_{2}}\right)(0,0)$ is simply the weighted average value of $e^{i k \phi_{2}}$ through the analysis window centered at $(\xi, \eta)$, the argument being approximately equal to $w * \phi_{2}(\xi, \eta)$, see [20].

The argument of $c_{f M, 0}$ can eventually be written as:

$$
\arg \left(d_{1}\right)+w * \phi_{2}(\xi, \eta)+\arg \left(1+\frac{d_{1} \mathcal{F}\left(e^{i k \phi_{2}}\right)(0,0)}{d_{k} \mathcal{F}\left(e^{i \phi_{2}}\right)(0,0)}\right)
$$

this equation holding modulo $2 \pi$. Now, the ratio $\mathcal{F}\left(e^{i k \phi_{2}}\right)(0,0) / \mathcal{F}\left(e^{i \phi_{2}}\right)(0,0)$ is a complex number of modulus approximately equal to 1 , and of argument $(1-k) w * \phi_{2}(\xi, \eta)$, which is small in the case of small displacements. Since, with the synthetic grids, $d_{1} / d_{k}$ is always a real number $\left(\ell(x)=\sin ^{7}(x)\right.$ being an odd function, its Fourier coefficients are all imaginary numbers), the last argument in (17) can be considered to be 0 . This discussion shows that, when an aliased spike is perfectly superposed to the principal harmonic, the resulting interference is negligible. This can be seen in the companion video when the grid pitch is set exactly to 6 pixels. Of course, this is only valid in the present case of synthetic grids: real grid profiles are likely to show asymmetries which prevent $\ell$ from being modeled as a strictly odd function.

\subsection{Real-case study}

A tensile test on a specimen equipped with two grids (one is aligned with the pixel grid, the other is rotated) was carried out to illustrate the benefit of rotated grids with respect to aliasing. An open-hole specimen in polymethylmethacrylate (PMMA) was considered here. The dimensions are $220 \times 50 \times 4 \mathrm{~mm}^{3}$. The diameter of the hole is $4 \mathrm{~mm}$. This hole was drilled to cause a heterogeneous strain distribution to locally appear in the specimen. The two grids were transferred on both sides 


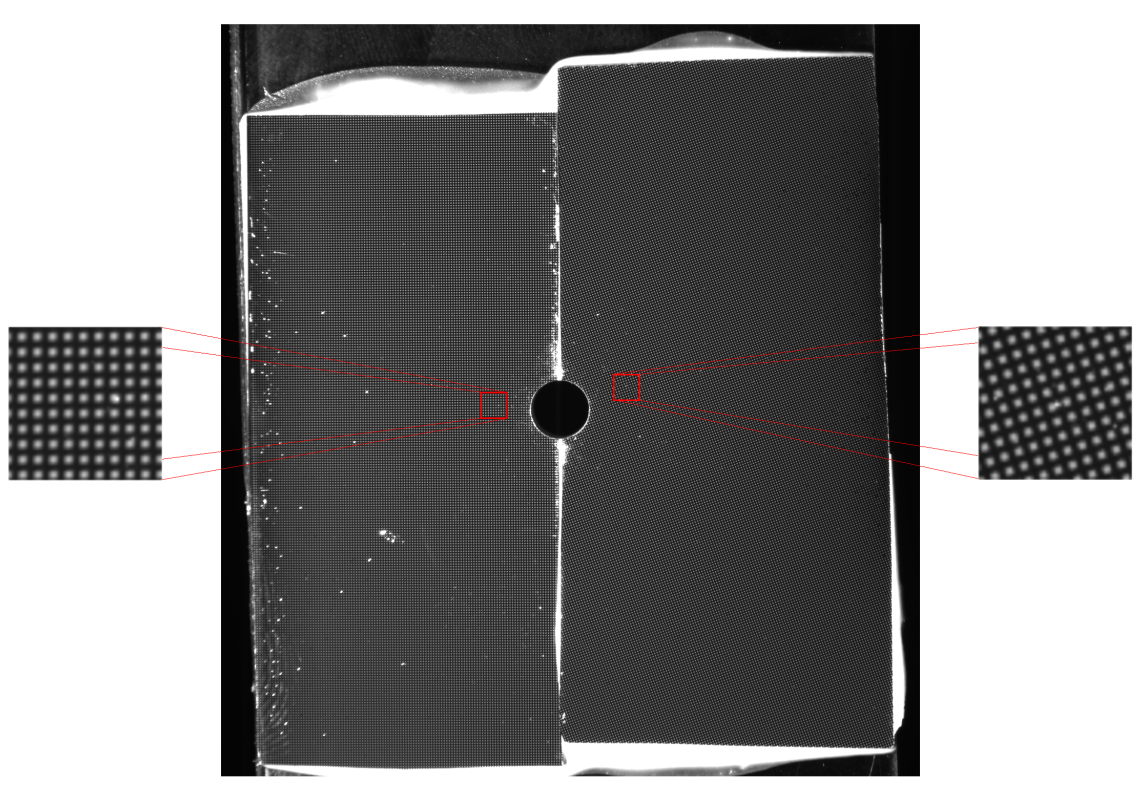

Figure 6: Real-case study. The two grids on a sample.

of the hole following the procedure proposed in [18] (see also [10]), as illustrated in Figure 6. The nominal pitch of these grids was $0.2 \mathrm{~mm}$. On the right-hand side of the hole, the grid was rotated by 19.5 degrees while the lines of the grid were parallel to the borders of the specimen on the left-hand side. The specimen was then subjected to a tensile test with a tensile machine. It can be checked that the material did not plastically deform with the corresponding stress level. A Sensicam QE 12-bit CCD camera shot the images of both grids while testing. The distance between specimen and camera was such that $p=5$ pixels were used to encode one grid period. These grid images were then processed separately to retrieve the phase distribution in each case, and then to deduce the $\varepsilon_{x x}$ strain distribution by using the procedure described in Section 2.3 above ( $x$ represents the horizontal direction in Figure 6).

One can notice that, with a small number of pixels per period (typically 5 pixels per period), a rotation of the imaged grid makes the contrast to slightly decrease, as peaks of the line profile are not aligned any more with the pixel grid but instead cross several pixels. As a consequence, the noise floor increases in displacement and strain maps, as explained in [9]. A larger number of pixels per period should circumvent this detrimental effect, as the sharpness of the image is likely to increase in this case.

As mentioned in Section 3, pixel intensity at the crossing of the lines is not exactly twice as high as the intensity of the line. This is not crucial since this model is accurate enough to build predictive formula which have been experimentally assessed in [9]. This explains, however, that the spectrum of a real grid image is not exactly as the one of the numerical simulation shown in Figure 5. A more accurate model of the continuous grid would be to enhance (6) with an additive term $B * \sum_{k, l} \delta_{k p, l p}$, where $B$ is the corrective intensity at the crossing of any two grid lines, approximately lying at pixels $(k p, l p)$. The Fourier transform of $s$ becomes (8), to which the 

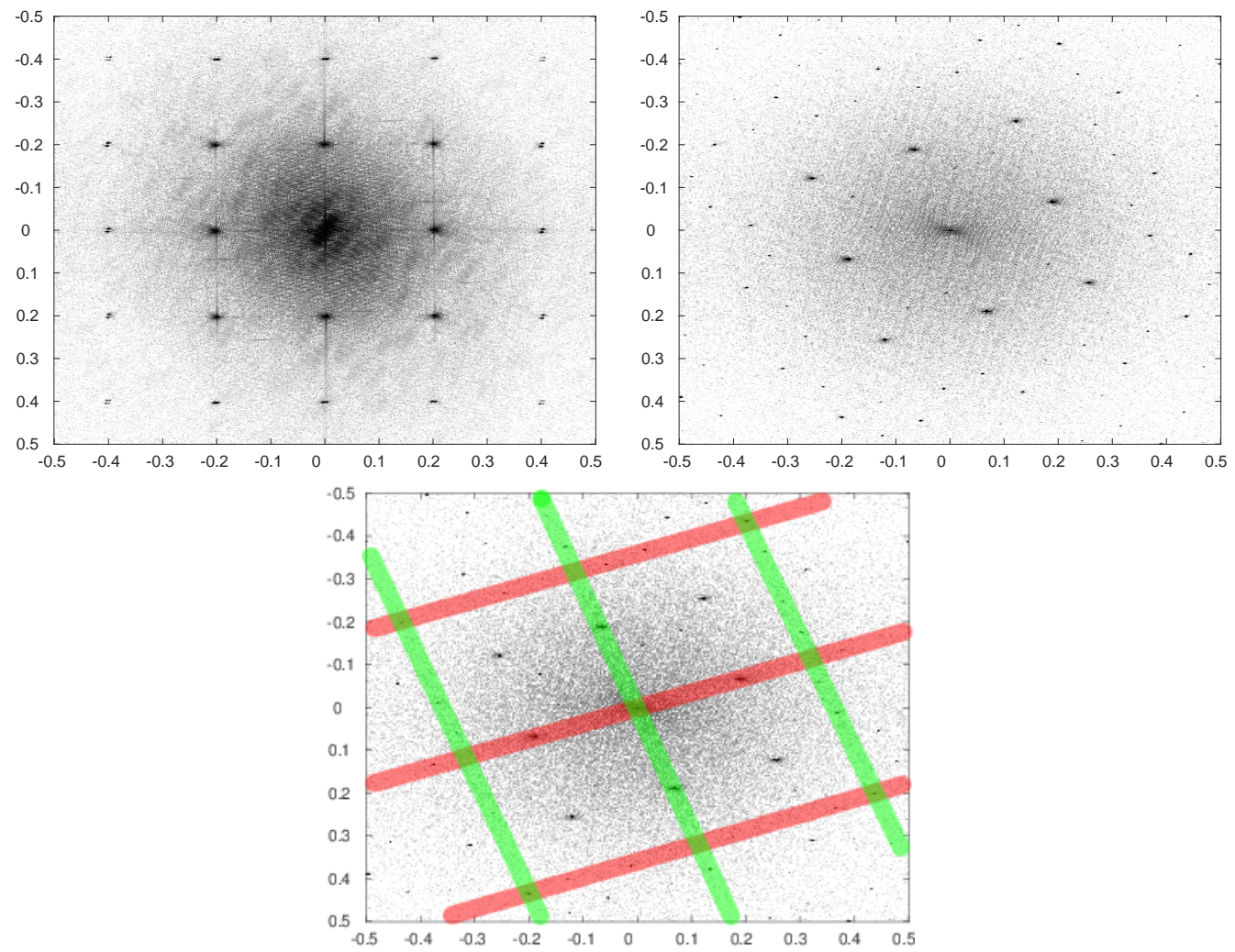

Figure 7: Real-case study. Spectra of the aligned-to-pixel grid (top-left) and of the rotated grid (top-right). Frequencies are in cycles per pixel. As can be seen in the bottom figure, aliased spikes are actually present in the spectrum of the aligned-to-pixel grid. These aliased spikes are shifted outside the principal harmonic when rotating the grid: in the annotated bottom spectrum, the aliased spikes are distributed in the straight lines on either sides of the middle line. In the aligned-to-pixel grid, the aliased spikes are almost superposed to the non-aliased ones.

following term is added:

$$
\mathcal{F}\left(B * \sum_{k, l} \delta_{k p, l p}\right)=f^{2} \mathcal{F}(B) \sum_{k, l} \delta_{k f, l f}
$$

The Fourier transform of the 2D Dirac comb $\sum_{k, l} \delta_{k p, l p}$ is indeed $f^{2} \sum_{k, l} \delta_{k f, l f}$.

Figure 6 shows the two grids on the sample. The spectra of the aligned-to-pixel grid and of the rotated grid are shown in Figure 7 . The spikes observed around the frequencies $(k f, l f)$ are consistent with the previous discussion. The frequencies around $(f, 0)$ and $(0, f)$ being covered by aliased spikes in the aligned-to-pixel grid, aliasing is expected to impair the retrieved strain map in this case. On the contrary, the aliased spikes do not seem to cover these frequencies in the spectrum of the rotated grid. The respective strain maps are shown in Figure 8. As expected from the aspect of the spectrum, aliasing affects the strain maps obtained from the aligned-to-pixel grids, and not 

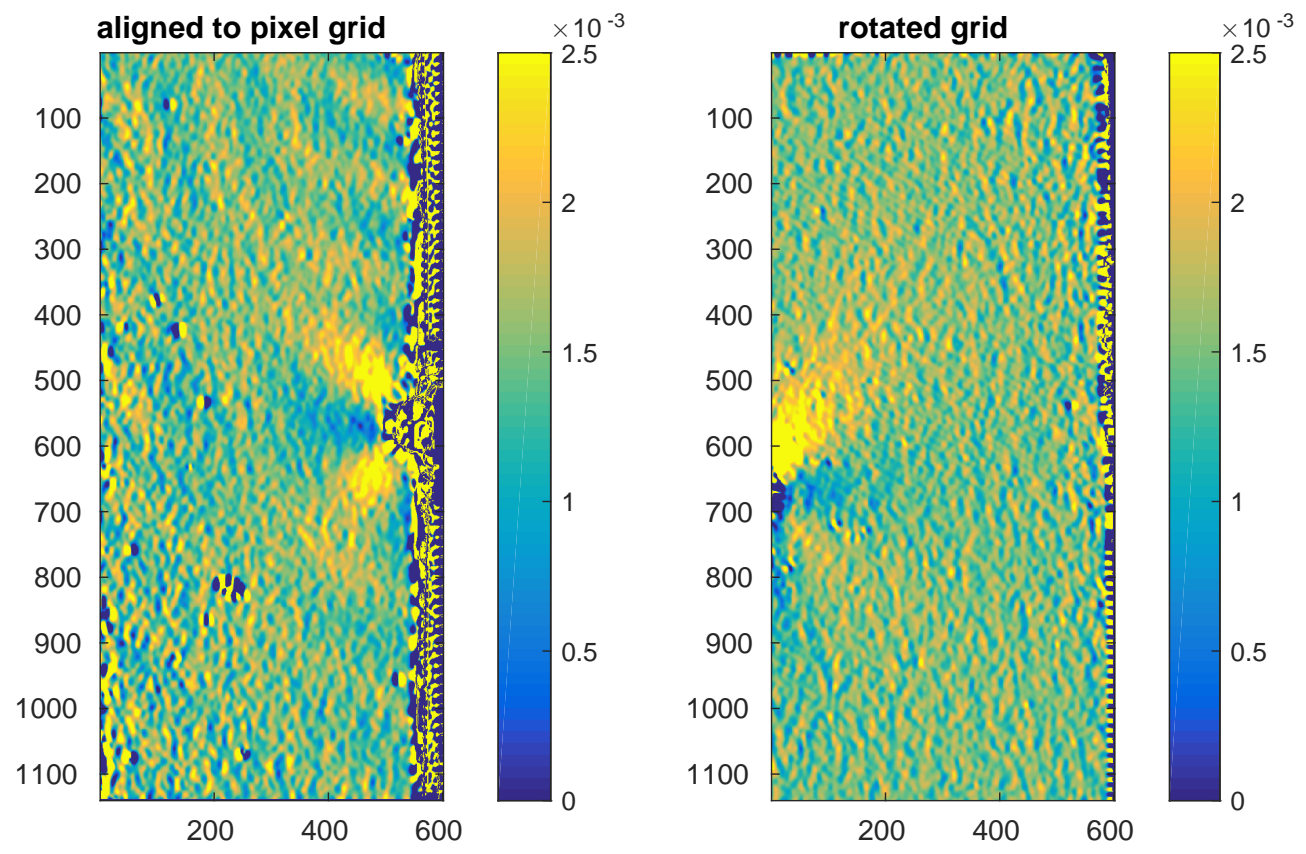

Figure 8: Real-case study. Retrieved strain map $\varepsilon_{x x}$ from the aligned-to-pixel grid (on the left) and from the rotated grid (on the right). The large parasitic fringes that can be seen on the left are not present on the right. These fringes are caused by aliasing. Note the effect of the noise, inherent to any digital acquisition device.

those obtained from the rotated grids.

The reader is invited to watch the second and third companion videos (available in supplementary files video_aliasing_real_epsxx.avi and video_aliasing_real_epsxy.avi). It can be seen that the strain distributions provided by the aligned-to-pixel grid are impaired by parasitic fringes, while those provided by the inclined one are not. The effect of aliasing is even stronger in the strain field increment. These fringes scroll while testing (thus as the load increases), which is due to the fact that the actual grid pitch slightly changes from one image to another because of the deformation of the specimen. In particular, the fringes disturb the actual strain distribution around the hole. The "flashing dots" correspond to the spatially correlated noise caused by sensor noise, which propagates from the images to the final strain maps whatever the orientation of the grid.

\section{Towards an optimum rotation angle}

The discussion of Section 3.2 tells us that if the line profile shows many harmonics of significant amplitude, then aliasing is mostly unavoidable. Nevertheless, if we assume that the line profile is made of only a few harmonics, then the corresponding spikes sparsely populate the discrete spectrum. It is possible to prevent aliased spikes to cover the principal harmonic by rotating the 
grid, as illustrated in Section 4 above. Intuitively speaking, we would like to set the rotation angle in such a way that the aliased spikes are as much as possible separated from the principal harmonic.

Let $\theta$ be the rotation angle. Thanks to the covariance property reminded in Section 2.2, the principal frequencies are thus $(f \cos (\theta),-f \sin (\theta))$ (to retrieve $\phi_{1}$ in $\left.(6)\right)$ and $(f \sin (\theta), f \cos (\theta))($ to retrieve $\left.\phi_{2}\right)$. Without loss of generality, we focus on $(f \cos (\theta),-f \sin (\theta))$, and assume that $0 \leq$ $\theta \leq \pi / 2$. The aliased harmonics that are the most likely to interfere with this frequency come from the nearest copies of $\mathcal{F}(s)$ to the principal frequencies, that is the ones translated from $(1,0)$ and $(0,-1)$ in $(15)$. The discussion of Section 4.2 shows that spikes from these translated copies are expected to be centered at

$$
(k f \cos (\theta)+l f \sin (\theta)+1,-k f \sin (\theta)+l f \cos (\theta))
$$

and

$$
\left(k^{\prime} f \cos (\theta)+l^{\prime} f \sin (\theta),-k^{\prime} f \sin (\theta)+l^{\prime} f \cos (\theta)-1\right)
$$

respectively, for any integer values $k, l$.

If $k, l$ had real values, the center of an aliased spike would be superposed to the principal harmonic provided that:

$$
\left\{\begin{array}{l}
f \cos (\theta)=k f \cos (\theta)+l f \sin (\theta)+1 \\
-f \sin (\theta)=-k f \sin (\theta)+l f \cos (\theta)
\end{array}\right.
$$

which gives after solving the linear system:

$$
\left\{\begin{array}{l}
k=1-\cos (\theta) / f \\
l=-\sin (\theta) / f
\end{array}\right.
$$

The nearest aliased spike to the principal frequency, among the copy of $\mathcal{F}(s)$ translated from $(1,0)$, is therefore such that:

$$
\left\{\begin{array}{l}
k=1-[\cos (\theta) / f] \\
l=-[\sin (\theta) / f]
\end{array}\right.
$$

where $[x]$ rounds $x$ to the nearest integer.

The same calculation shows that the nearest aliased spike to the principal frequency, among the copy of $\mathcal{F}(s)$ translated from $(0,-1)$, is such that:

$$
\left\{\begin{array}{l}
k^{\prime}=1-[\sin (\theta) / f] \\
l^{\prime}=[\cos (\theta) / f]
\end{array}\right.
$$

In the first case, the squared distance between the principal harmonic and an aliased spike is

$$
\begin{aligned}
D_{f}^{2}(\theta)= & (k f \cos (\theta)+l f \sin (\theta)+1-f \cos (\theta))^{2}+(-k f \sin (\theta)+l f \cos (\theta)+f \sin (\theta))^{2} \\
= & (k-1)^{2} f^{2} \cos ^{2}(\theta)+l^{2} f^{2} \sin ^{2}(\theta)+1+2(k-1) l f^{2} \cos (\theta) \sin (\theta)+2(k-1) f \cos (\theta) \\
& \quad+2 l f \sin (\theta)+(k-1)^{2} f^{2} \sin ^{2}(\theta)+l^{2} f^{2} \cos ^{2}(\theta)-2(k-1) l f^{2} \cos (\theta) \sin (\theta) \\
= & (k-1)^{2} f^{2}+2(k-1) f \cos (\theta)+1+l^{2} f^{2}+2 l f \sin (\theta)
\end{aligned}
$$

This expression simplifies into $((k-1) f+\cos (\theta))^{2}+(l f+\sin (\theta))^{2}$.

In the second case (to retrieve $\left.\phi_{2}\right)$, the same calculation gives $\left(\left(k^{\prime}-1\right) f+\sin (\theta)\right)^{2}+\left(l^{\prime} f-\cos (\theta)\right)^{2}$.

With $(k, l)$ as in $(23)$ and $\left(k^{\prime}, l^{\prime}\right)$ as in $(24)$, these two squared distances are identical and we can conclude that the squared distance between the nearest aliased spike and the principal frequency is simply:

$$
D_{f}^{2}(\theta)=f^{2}\left(\{\cos (\theta) / f\}^{2}+\{\sin (\theta) / f\}^{2}\right)
$$




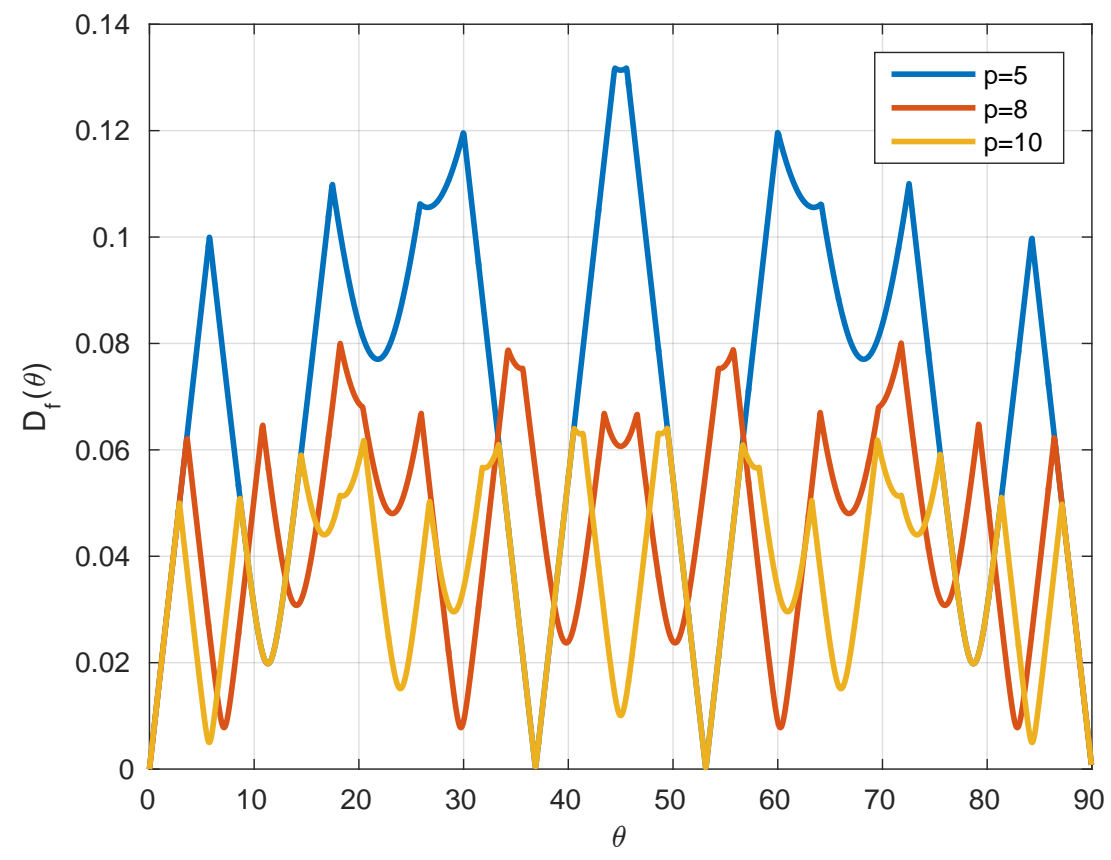

Figure 9: Plot of $D_{f}(\theta)$ against $\theta \in\left[0,90^{\circ}\right]$ for three typical values of the grid pitch $p=1 / f$.

where $\{x\}=x-[x]$ denotes the distance between any real number $x$ and the nearest integer to $x$.

Figure 9 shows three plots of $D_{f}(\theta)$ for three typical values of the grid pitch $p=1 / f(5,8$, and 10 pixels). For example, for $p=1 / f=5$ pixels, an angle of $45^{\circ}$ is optimum, but an angle of $30^{\circ}$ is still appropriate. It is interesting to note that, with $p=10$ pixels, an angle of $45^{\circ}$ is not appropriate and an angle of $40^{\circ}$ should be preferred. As we can see, the value of the maxima of $D_{f}$ decreases as $p$ increases. This is logical since the spikes are more densely spread over the spectrum as $p$ increases, the distance between the principal frequency and the nearest aliased spike thus decreases. Plotting (28) gives an indication on how to set the rotation angle, by choosing an angle maximizing $D_{f}$. However, it may be practically more convenient to use a local maximum corresponding to a wide peak instead of the global maximum, which may correspond to a sharp peak. With a sharp peak, a small error on the angle is indeed likely to give aliasing. Moreover, the user shall not forget that only "first order" aliased spikes are considered in the present calculation, spikes coming from translation of the continuous spectrum larger than 2 in (15) are not considered. This simplification may be over-optimistic if the grid profile is not smooth enough, which would prevent the Fourier coefficients $d_{k}$ from quickly vanishing and would produce many harmonics with a significant amplitude. In addition, it should be mentioned that the spreading of the spectrum spikes is not taken into account in the preceding discussion: moving the center of the aliased spikes away from the principal frequency may not be sufficient because of the width of the aliased spikes which may be too large. 


\section{Conclusion}

In this paper, we analyzed spatial aliasing in the context of grid images acquired through a digital camera, and the resulting effect on the displacement and strain maps estimated from these images. The discussion is independent from the spectral technique used, even though the WFT set on the nominal frequency of the grid was employed here. We have proved in a theoretical model that rotating the grid permits to reduce aliasing. This approach was assessed both in a numerical study and in a real experiment. We have also proposed a way to optimally set the rotation angle so that the aliased spikes are as much as possible separated from the principal harmonic.

Acknowledgments. This work is partially funded by GdR CNRS ISIS (Timex project). The authors acknowledge useful discussions with Johan Hoefnagels. They also thank Mohamed Cheikh Teguedi for his assistance in the experimental part of the study.

\section{A Spectrum of a rotated image}

Let $\theta \in \mathbb{R}$ and $R_{\theta}=\left(\begin{array}{cc}\cos (\theta) & \sin (\theta) \\ -\sin (\theta) & \cos (\theta)\end{array}\right)$ be the corresponding rotation matrix. For any 2D function $f$ representing a continuous image, the Fourier transform of the rotated image writes:

$$
\mathcal{F}\left(f \circ R_{\theta}\right)(\mathbf{u})=\iint_{\mathbb{R}^{2}} f\left(R_{\theta} \mathbf{x}\right) e^{-2 i \pi<\mathbf{x}, \mathbf{u}>} \mathrm{d} \mathbf{x}
$$

where $\mathbf{u} \in \mathbb{R}^{2},\langle\cdot, \cdot\rangle$ is the standard scalar product, and $\circ$ denotes the composition between functions. A change of variable gives

$$
\mathcal{F}\left(f \circ R_{\theta}\right)(\mathbf{u})=\iint_{\mathbb{R}^{2}} f(\mathbf{x}) e^{-2 i \pi<R_{\theta}^{-1} \mathbf{x}, \mathbf{u}>} \mathrm{d} \mathbf{x}
$$

Since any rotation is a unitary transform, we deduce $\left.\left\langle R_{\theta}^{-1} \mathbf{x}, \mathbf{u}\right\rangle=<\mathbf{x}, R_{\theta} \mathbf{u}\right\rangle$, therefore (4) holds.

Concerning the windowed Fourier transform, the same argument gives

$$
\begin{aligned}
\mathcal{F}_{w}\left(f \circ R_{\theta}\right)(\xi, \mathbf{u}) & =\iint_{\mathbb{R}^{2}} f\left(R_{\theta} \mathbf{x}\right) w(\mathbf{x}-\xi) e^{-2 i \pi<\mathbf{x}, \mathbf{u}>} \mathrm{d} \mathbf{x} \\
& =\iint_{\mathbb{R}^{2}} f(\mathbf{x}) w\left(R_{\theta}^{-1} \mathbf{x}-\xi\right) e^{-2 i \pi<\mathbf{x}, R_{\theta} \mathbf{u}>} \mathrm{d} \mathbf{x}
\end{aligned}
$$

The analysis window being isotropic, $w\left(R_{\theta}^{-1} \mathbf{x}-\xi\right)=w\left(R_{\theta}^{-1}\left(\mathbf{x}-R_{\theta} \xi\right)\right)=w\left(\mathbf{x}-R_{\theta} \xi\right)$, therefore (5) holds.

\section{B Spectrum of a continuous grid}

Let us recall that, with the definition of the Fourier transform given in (1), the following relations hold for tempered distributions:

$$
\begin{aligned}
\mathcal{F}(1) & =\delta_{0,0} \\
\mathcal{F}\left(e^{-2 \pi i(a x+b y)}\right) & =\delta_{a, b} \\
\mathcal{F}(f \cdot g) & =\mathcal{F}(f) * \mathcal{F}(g)
\end{aligned}
$$


where $\delta_{a, b}$ is the Dirac delta function concentrated at $(a, b) \in \mathbb{R}^{2}$.

With this formula, the Fourier transform of (6) writes:

$$
\begin{gathered}
\mathcal{F}(s)=\mathcal{F}(A)+\frac{\gamma}{2} \sum_{k \in \mathbb{Z}} d_{k}\left(\mathcal{F}\left(A(x, y) e^{2 i \pi k f x+i k \phi_{1}(x, y)}\right)+\mathcal{F}\left(A(x, y) e^{2 i \pi k f y+i k \phi_{2}(x, y)}\right)\right) \\
=\mathcal{F}(A)+\frac{\gamma}{2} \mathcal{F}(A) *\left(\sum_{k \in \mathbb{Z}} d_{k} \mathcal{F}\left(e^{2 \pi i f k x}\right) * \mathcal{F}\left(e^{i k \phi_{1}}\right)\right) \\
+\frac{\gamma}{2} \mathcal{F}(A) *\left(\sum_{k \in \mathbb{Z}} d_{k} \mathcal{F}\left(e^{2 \pi i f k y}\right) * \mathcal{F}\left(e^{i k \phi_{2}}\right)\right)
\end{gathered}
$$

Consequently,

$\mathcal{F}(s)(\xi, \eta)=\mathcal{F}(A)(\xi, \eta)+\frac{\gamma}{2} \sum_{k \in \mathbb{Z}} d_{k} \mathcal{F}(A) * \mathcal{F}\left(e^{i k \phi_{1}}\right)(\xi-k f, \eta)+\frac{\gamma}{2} \sum_{k \in \mathbb{Z}} d_{k} \mathcal{F}(A) * \mathcal{F}\left(e^{i k \phi_{2}}\right)(\xi, \eta-k f)$

Indeed, $\mathcal{F}\left(e^{2 \pi i f k x}\right)=\delta_{-k f, 0}$.

In practice, the illumination field $A$ varies slowly across the imaged grid, giving a concentrated spectrum around the mean value $\mathcal{F}(A)(0,0)$. In the limit-case of a constant illumination $A(x, y)=$ $A, \mathcal{F}(A)=A \delta_{0,0}$, and the preceding formula simplifies into

$$
\mathcal{F}(s)(\xi, \eta)=A \delta_{0,0}(\xi, \eta)+\frac{\gamma A}{2} \sum_{k \in \mathbb{Z}} d_{k} \mathcal{F}\left(e^{i k \phi_{1}}\right)(\xi-k f, \eta)+\frac{\gamma A}{2} \sum_{k \in \mathbb{Z}} d_{k} \mathcal{F}\left(e^{i k \phi_{2}}\right)(\xi, \eta-k f)
$$

This proves (8) and (9).

\section{Stationary phase method}

A simplified evaluation of $\mathcal{F}\left(e^{i \psi}\right)$ (with the notations of Section $3, \psi=k \phi_{1}$ or $=k \phi_{2}$ ) is available through the stationary phase method, which we briefly recall here. The basic idea of this method is that sine functions with a quickly varying phase over a given region integrates to 0 on this region. Since

$$
\mathcal{F}\left(e^{i \psi}\right)(u, v)=\iint_{\mathbb{R}^{2}} e^{i \psi(x, y)-2 i \pi(x u+y v)} \mathrm{d} x \mathrm{~d} y
$$

this principle justifies that the only significant contribution is caused by regions in $\mathbb{R}^{2}$ where the phase $\varphi(x, y)=\psi(x, y)-2 \pi(x u+y v)$ does not oscillate (hence "stationary phase"), that is, where the partial derivatives of the phase $\varphi$ are zero.

Consequently, the Fourier transform of $e^{i \psi}$ can be approximated by

$$
\mathcal{F}\left(e^{i \psi}\right)(u, v) \simeq \sum_{\left(x_{i}, y_{i}\right)} \int_{x_{i}-\varepsilon}^{x_{i}+\varepsilon} \int_{y_{i}-\varepsilon}^{y_{i}+\varepsilon} e^{i \psi(x, y)-2 i \pi(x u+y v)} \mathrm{d} x \mathrm{~d} y
$$

where the $\left(x_{i}, y_{i}\right)$ are such that $\partial \psi / \partial x\left(x_{i}, y_{i}\right)=2 \pi u$ and $\partial \psi / \partial y\left(x_{i}, y_{i}\right)=2 \pi v$, and $\varepsilon>0$ defines the half-width of the (small) region giving the contribution to the integral over $\mathbb{R}^{2}$. Under the same principle, $\mathcal{F}\left(e^{i \psi}\right)(u, v)=0$ if $2 \pi u$ (resp. $2 \pi v$ ) is not within the range of the values taken by $\partial \psi / \partial x$ (resp. $\partial \psi / \partial y$ ), which means that the phase derivatives cannot vanish. 
Approximating $\psi(x, y)-2 \pi(x u+y v)$ as a function of $(x, y)$ by its second-order Taylor expansion around $\left(x_{i}, y_{i}\right)$ gives, the gradient being null at these points:

$$
\psi(x, y)-2 \pi(x u+y v) \simeq \psi\left(x_{i}, y_{i}\right)-2 \pi\left(x_{i} u+y_{i} v\right)+\frac{1}{2}\left(x-x_{i}, y-y_{i}\right) H_{\psi}\left(x_{i}, y_{i}\right)\left(x-x_{i}, y-y_{i}\right)^{T}
$$

where $H_{\psi}\left(x_{i}, y_{i}\right)$ is the $2 \times 2$ Hessian matrix of $\psi$ at $\left(x_{i}, y_{i}\right)$. Plugging (42) into (41) and substituting in the integral $x-x_{i}$ by $x$ and $y-y_{i}$ by $y$ gives:

$$
\mathcal{F}\left(e^{i \psi}\right)(u, v) \simeq \sum_{\left(x_{i}, y_{i}\right)} e^{i \pi \psi\left(x_{i}, y_{i}\right)-2 i \pi\left(x_{i} u+y_{i} v\right)} \int_{-\varepsilon}^{\varepsilon} \int_{-\varepsilon}^{\varepsilon} e^{i\left(\frac{1}{2}(x, y) H_{\psi}\left(x_{i}, y_{i}\right)(x, y)^{T}\right)} \mathrm{d} x \mathrm{~d} y
$$

The domain of the preceding integrals can be extended to $\mathbb{R}^{2}$, if $H_{\psi}$ is non-degenerated, through the stationary phase principle (the contribution of the terms outside $[-\varepsilon, \varepsilon] \times[-\varepsilon, \varepsilon]$ being negligible because of the quickly varying phase), which gives:

$$
\begin{aligned}
\mathcal{F}\left(e^{i \psi}\right)(u, v) & \simeq \sum_{\left(x_{i}, y_{i}\right)} e^{i \pi \psi\left(x_{i}, y_{i}\right)-2 i \pi\left(x_{i} u+y_{i} v\right)} \iint_{\mathbb{R}^{2}} e^{i\left(\frac{1}{2}(x, y) H_{\psi}\left(x_{i}, y_{i}\right)(x, y)^{T}\right)} \mathrm{d} x \mathrm{~d} y \\
& =\sum_{\left(x_{i}, y_{i}\right)} \frac{2 \pi e^{i \sigma\left(H_{\psi}\left(x_{i}, y_{i}\right)\right) \pi / 4}}{\left|\operatorname{det} H_{\psi}\left(x_{i}, y_{i}\right)\right|^{1 / 2}} e^{i \pi \psi\left(x_{i}, y_{i}\right)-2 i \pi\left(x_{i} u+y_{i} v\right)}
\end{aligned}
$$

where $\operatorname{det}(A)$ denotes the determinant of any square matrix $A$ and $\sigma(A)$ is the sign of $\operatorname{det} A$, since the integral over $\mathbb{R}^{2}$ in (44) is a multidimensional Fresnel integral.

If no stationary point of the phase in (41) exists, which means that the system

$$
\left\{\begin{array}{l}
\frac{\partial \psi}{\partial x}(x, y)-2 \pi u=0 \\
\frac{\partial \psi}{\partial y}(x, y)-2 \pi v=0
\end{array}\right.
$$

has no solution in $(x, y)$, we obtain, for any $u, v$,

$$
\mathcal{F}\left(e^{i \psi}\right)(u, v) \simeq 0
$$

In other words, we have proved the following proposition.

Proposition C.1 If the absolute value of the derivatives $\partial \Psi / \partial x$ and $\partial \Psi / \partial y$ are bounded from above by $B^{x}$ and $B^{y}$ respectively, then for any $(u, v)$ such that $|u|>B^{x} /(2 \pi)$ and $|v|>B^{y} /(2 \pi)$, $\mathcal{F}\left(e^{i \Psi}\right)(u, v)=0$.

This means that the support of $\mathcal{F}\left(e^{i \Psi}\right)$ is included in $\left[-\frac{B^{x}}{2 \pi}, \frac{B^{x}}{2 \pi}\right] \times\left[-\frac{B^{y}}{2 \pi}, \frac{B^{y}}{2 \pi}\right]$.

\section{Link between discrete spectrum and Fourier transform of a continuous $2 \mathrm{D}$ signal}

Since $\bar{s}(k, l)=s(k, l)$ and $s(x, y)=0$ as soon as $(x, y) \notin[-M / 2, M / 2-1] \times[-N / 2, N / 2-1]$,

$$
\begin{aligned}
c_{m, n} & =\sum_{k=-M / 2}^{M / 2-1} \sum_{l=-N / 2}^{N / 2-1} \bar{s}(k, l) e^{-2 i \pi(k m / M+l n / N)} \\
& =\sum_{(k, l) \in \mathbb{Z}^{2}} s(k, l) e^{-2 i \pi(k m / M+l n / N)}
\end{aligned}
$$


We obtain the following expression with the Poisson summation formula (under mild assumptions on $f, \sum_{(k, l) \in \mathbb{Z}^{2}} f(k, l)=\sum_{(k, l) \in \mathbb{Z}^{2}} \mathcal{F}(f)(k, l)$ holds) and the property of the Fourier transform of a translated function reminded in Appendix B:

$$
\begin{aligned}
c_{m, n} & =\sum_{(k, l) \in \mathbb{Z}^{2}} \mathcal{F}\left(s e^{-2 i \pi(\cdot m / M+\cdot n / N)}\right)(k, l) \\
& =\sum_{(k, l) \in \mathbb{Z}^{2}} \mathcal{F}(s)(k+m / M, l+n / N)
\end{aligned}
$$

which is (14).

\section{References}

[1] I. Aizenberg and C. Butakoff. Frequency domain median-like filter for periodic and quasiperiodic noise removal. In SPIE Proceedigns of Image Processing: Algorithms and Systems, volume 4767, pages 181-191, 2002.

[2] I. Aizenberg and C. Butakoff. A windowed Gaussian notch filter for quasi-periodic noise removal. Image and Vision Computing, 26(10):1347-1353, 2008.

[3] L. Allais, M. Bornert, T. Bretheau, and D. Caldemaison. Experimental characterization of the local strain in a heterogeneous elastoplastic material. Acta Metallurgica et Materialia, 42(11):3865-3880, 1994.

[4] C. Badulescu, M. Grédiac, and J.-D. Mathias. Investigation of the grid method for accurate in-plane strain measurement. Measurement Science and Technology, 20(9):095102, 2009.

[5] C. Badulescu, M. Grédiac, J.-D. Mathias, and D. Roux. A procedure for accurate onedimensional strain measurement using the grid method. Experimental Mechanics, 49(6):841$854,2009$.

[6] X. Dai, H. Xie, H. Wang, C. Li, Z. Liu, and L. Wu. The geometric phase analysis method based on the local high resolution discrete Fourier transform for deformation measurement. Measurement Science and Technology, 25(2):025402, 2014.

[7] X. Dai, H. Xie, and Q. Wang. Geometric phase analysis based on the windowed Fourier transform for the deformation field measurement. Optics and Laser Technology, 58:119-127, 2014.

[8] X. Du, B.W. Anthony, and N.C. Kojimoto. Grid-based matching for full-field large-area deformation measurement. Optics and Lasers in Engineering, 66:307-319, 2015.

[9] M. Grédiac and F. Sur. Effect of sensor noise on the resolution and spatial resolution of displacement and strain maps estimated with the grid method. Strain, 50(1):1-27, 2014.

[10] M. Grédiac, F. Sur, and B. Blaysat. The grid method for in-plane displacement and strain measurement: a review and analysis. Strain, 52(3):205-243, 2016.

[11] M. Grédiac, F. Sur, and B. Blaysat. Removing quasi-periodic noise in strain maps by filtering in the Fourier domain. Experimental Techniques, 40(3):959-971, 2016. 
[12] G.A.A. Hudhud and M.J. Turner. Digital removal of power frequency artifacts using a Fourier space median filter. IEEE Signal Processing Letters, 12(8):573-576, 2005.

[13] M.J. Hÿtch, E. Snoeck, and R. Kilaas. Quantitative measurement of displacement and strain fields from HREM micrographs. Ultramicroscopy, 74(3):131-146, 1998.

[14] G.-M. Ionita, D. Coltuc, S. G. Stanciu, and D. E. Tranca. Automatic moiré pattern removal in microscopic images. In Proceedings of the 19th International Conference on System Theory, Control and Computing (ICSTCC), pages 776-779, Cheile Gradistei, Romania, 2015.

[15] JCGM member organizations. International vocabulary of metrology. basic and general concepts and associated terms (VIM), 2008.

[16] S. Mallat. A wavelet tour of signal processing (2nd edition). Academic Press, 1999.

[17] D.P. Petersen and D. Middleton. Sampling and reconstruction of wave-number-limited functions in $N$-dimensional Euclidean spaces. Information and Control, 5:279-323, 1962.

[18] J.L. Piro and M. Grédiac. Producing and transferring low-spatial-frequency grids for measuring displacement fields with moiré and grid methods. Experimental Techniques, 28(4):23-26, 2004.

[19] F. Sur. An a-contrario approach to quasi-periodic noise removal. In Proceedings of the IEEE International Conference on Image Processing (ICIP), pages 3841-3845, Québec City, Canada, 2015.

[20] F. Sur and M. Grédiac. Towards deconvolution to enhance the grid method for in-plane strain measurement. AIMS Inverse Problems and Imaging, 8(1):259-291, 2014.

[21] F. Sur and M. Grédiac. Automated removal of quasiperiodic noise using frequency domain statistics. ISET / SPIE Journal of Electronic Imaging, 24(1):013003/1-19, 2015.

[22] F. Sur and M. Grédiac. Influence of the analysis window on the metrological performance of the grid method. Journal of Mathematical Imaging and Vision, 2016. To be published.

[23] Y. Surrel. Photomechanics, volume 77 of Topics in Applied Physics, chapter Fringe analysis, pages 55-102. Springer, 2000.

[24] M. Sutton, J.-J. Orteu, and H. Schreier. Image Correlation for Shape, Motion and Deformation Measurements. Springer, 2009.

[25] M. Takeda. Spatial-carrier fringe-pattern analysis and its applications to precision interferometry and profilometry: An overview. Industrial Metrology, 1(2):79-99, 1990.

[26] M. Takeda and K. Mutoh. Fourier transform profilometry for the automatic measurement of 3-d object shapes. Applied Optics, 22(24):3977-3982, 1983.

[27] J. Wang and D.C. Liu. 2D FFT for periodic noise removal on strain images. In Proceedings of the 4th International Conference on Bioinformatics and Biomedical Engineering (ICBBE), Chengdu, China, 2010. IEEE.

[28] Z. Wei, J. Wang, H. Nichol, S. Wiebe, and D. Chapman. A median-Gaussian filtering framework for moiré pattern noise removal from X-ray microscopy image. Micron: the international research and review journal for microscopy, 43(2-3):170-176, 2012. 